was seen about the time the blast wave should have crossed the magnetic field line intersecting the payload. Nearly coincident with this, electron fluxes of $2-k \mathrm{~V}$ were seen moving up and down the field lines, but the electrons did not actually seem to reach the atmosphere. The currents generated by the electric field apparently set up reflecting electric shocks both above and below the burst point, and the electrons were probably trapped between them.

The discrete auroras are believed to be caused by electrons accelerated through similar shocks, or double layers, also set up by currents flowing parallel to magnetic field lines; but although the current-driven shocks play roles in both the natural aurora and the Trigger experiment, their roles are quite different.

The instrument package also observed electron fluxes with energies greater than 40 kiloelectron volts (keV). There was a prompt burst followed a second later by sustained fluxes of electrons. Fluxes greater than $40 \mathrm{keV}$ are believed to be responsible for the patchy and pulsating aurora seen in the early morning hours in the diffuse aurora (see review by A.D. Johnstone Nature 119, 274; 1978). The natural precipitation is also accompanied by electromagnetic wave emissions called chorus. The chorus emission is in the audio frequency range, 0.5 to $2 \mathrm{kHz}$, and when amplified sounds like the chirping of birds. The precipitation can be enhanced by increasing the ambient ionization because the ambient ionization makes the trapped electrons more unstable to precipitation caused by the electromagnetic emissions.

It is tempting to attribute the delayed precipitation of electrons of energy greater than $40 \mathrm{keV}$ in Trigger to the enhanced ionization density left by the caesium in the wake of the Trigger blast wave. The delayed fluxes were accompanied by wave emissions, but the emissions were electrostatic, rather than electromagnetic, and so would not probably be able to cause precipitation of trapped fluxes. Moreover, the Trigger fluxes were primarily field aligned, which is again inconsistent with a wave-particle diffusion process. The experimenters attribute most of the enhancement to noise contamination of the magnetic field-aligned detector. However, an X-ray detector suspended from a parachute that was deployed by a second rocket did observe $0.13-\mathrm{Hz}$ pulsations in $\mathrm{X}$-ray fluxes some two minutes after the event. This frequency is comparable with pulsation frequencies observed in natural aurora.

The prompt energetic electron fluxes are believed to be real, and they are accompanied by an electromagnetic pulse. However, as the electron fluxes are also primarily field aligned, the acceleration mechanism would have to be a stochastic process, since the electric potentials generated by the explosion are considerably less than the observed electron energies.

The Trigger experiment initiated a number of interesting plasma processes, some of which are similar to those responsible for the aurora. However, the natural aurora and the events observed in Trigger are not similar enough for the Trigger results to be applied in a direct way towards understanding the aurora; but after further theoretical interpretation it may turn out that the experiment has provided a number of useful clues to understanding processes in the natural aurora.

Certainly, the concept of the Trigger experiment is very appealing. Not only does the technique of artifically perturbing the ionosphere or magnetosphere allow the study of a wide range of general plasma processes, it also makes it possible to separate cause and effect, an often serious problem in the interpretation of auroral observations.

The use of chemical releases in space research will increase; groups from many countries are now proposing that such release experiments be conducted from a 'chemical release module' which will be be launched through the US space shuttle programme sometime in the mid-eighties.

\title{
Are membrane proteins introverted?
}

from N. Michael Green

ThE PURPLE MEMBRANE of Halobacterium 1979; Rogan and Zaccai J. molec. Biol. halobium has become a test bed for 145,$281 ; 1980$.).

sophisticated new techniques. These, in Purple membranes were prepared in turn, have given exciting new information which all of either the valine or the about the structure and function of the phenylalanine residues had been replaced major protein of the purple membrane, by their fully deuterated analogues and the bacterial rhodopsin. The most recent neutron diffraction patterns were comtechnique, neutron diffraction, shows that pared with that from normal membrane. rhodopsin is an "inside-out" protein. Six From the latter a neutron density map was years ago a combination of novel electron constructed which closely resembled the microscopic techniques with X-ray electron density map. Difference Fourier diffraction gave a picture of thestructure at maps relative to the deuterated $7 \AA$ resolution (Henderson and Unwin preparations were computed and showed Nature 257, 28; 1975). Then last year, a that valine and phenylalanine residues were correlation of the electron density concentrated in different regions. This was distribution with the amino acid sequence to be expected, since these residues have of appropriate helical segments produced a rather different distributions both between tentative model structure (Engelman et al. the seven transmembrane helices and Proc. natn. Acad. Sci. U.S.A. 77, 2023; azimuthally within each helix. The peak 1980). It is this model which now receives density differences for both valine and strong support from some elegant neutron phenylalanine side chains were off the helix diffraction experiments (Engelman and axes, as would be expected, but whereas the Zaccai Proc. natn. Acad. Sci. U.S.A. 77, valine peaks were displaced towards the $5894 ; 1980)$. This method, based on the exterior of the molecule those of great difference between neutron phenylalanine were nearer to the interior, scattering by protons and deuterons, has in the region where many of the charged also been used to show that there are no groups had been placed in the tentative detectable aqueous channels in the structure of Engelman et al. This membrane (Zaccai J. molec. Biol 132, 181; differential distribution was consistent with the relative azimuthal distributions of valine and phenylalanine derived from the N. Michael Green is in the National Institute for Medical Research, Mill Hill, London.

sequences of the previously assigned helices.
Comparison of the sequence-based azimuthal distributions with those of the charged residues confirms that valines (17 out of 19) are on the opposite sides of helices from phenylalanines ( 9 out of 11 ), and that the buried charged groups go with the phenylalanines. Moreover, the previously assigned locations of helices rich in valine or phenylalanine were consistent with the Fourier difference maps derived from neutron scattering, though the authors refrain from any detailed interpretation of this at the moment. The consistency of these new results with the earlier model is impressive and gives confidence in the validity of the arguments used in support of the previous assignments.

A firm picture is beginning to emerge of an 'inside-out' protein molecule with buried polar and charged groups located predominantly between helices and with most of the interface between lipid methylene groups and the protein being occupied by hydrophobic aliphatic chains. The authors suggest that in addition to a possible role in providing sites for proton translocation, the charged and polar groups would be an important stabilizing element in such a predominantly hydrophobic structure, since in their absence interactions between hydrophobic helices immersed in a hydrophobic solvent are very weak. 